\title{
A Comparative Study of Closure of Tympanic Membrane Perforation between Chemical Cauterization and Fat Plug Myringoplasty
}

\author{
Manabendra Debnath, Swagata Khanna \\ Department of ENT, Gauhati Medical College \& Hospital, Guwahati, India \\ Email: swagatakhanna@sify.com
}

Received July 13, 2013; revised August 15, 2013; accepted September 2, 2013

Copyright (C) 2013 Manabendra Debnath, Swagata Khanna. This is an open access article distributed under the Creative Commons Attribution License, which permits unrestricted use, distribution, and reproduction in any medium, provided the original work is properly cited.

\begin{abstract}
The purpose of closure of small dry tympanic membrane perforations is to restore the continuity of the tympanic membrane in order to improve hearing and decrease the incidence of middle ear infections. Small and cost effective procedures like Chemical Cauterization and Fat Plug Myringoplasty have been found to be effective in healing small central perforations with significant hearing improvement. A study was carried out in 69 patients with central perforations in the Department of ENT, Gauhati Medical College \& Hospital for the duration of 1 year from August 11 to July 12. Out of 69 patients, 36 underwent Chemical Cauterization and 33 Fat Plug Myringoplasty. Pre- and post-operative hearing assessments of each patient were done and statistically analyzed. The success rate was found to be $83.33 \%$ and $90.9 \%$ respectively. In both groups, there was statistically significant hearing improvement with a P value 0.01 . From our study, it was found that the two procedures are simple, reliable and also lessened the morbidity of the patient. Thus we recommend the use of these two official procedures for the treatment of central perforations of tympanic membrane less than $5 \mathrm{~mm}$.
\end{abstract}

Keywords: Fat Plug Myringoplasty; Chemical Cauterization; Paper Patching; Central Perforations

\section{Introduction}

Tympanic membrane perforations result mainly from infectious and traumatic etiologies. Perforations result from acute otitis media and trauma heal spontaneously in the majority of cases. But if there is repeated infections or if the infection is persistent, there is less possibility of spontaneous healing of these perforations. Long-standing tympanic membrane perforations may cause conductive hearing loss and middle ear infection even if they are small. The purpose of closure of chronic dry tympanic membrane perforations is to restore the continuity of the tympanic membrane in order to improve hearing and decrease the incidence of middle ear infections.

Myringoplasty is the procedure of closure of the perforations of the pars tensa of the tympanic membrane. The first attempt at myringoplasty was recorded by Marcus Banzer in 1640 [1]. The first surgical closure of tympanic membrane perforations using an autograft (a full thickness free skin graft) was performed by Berthold in 1878 and he introduced the term "Myringoplasty" [1]. Although Tympanoplasty (myringoplasty + ossicular chain reconstruction) is the ideal treatment, simple and cost effective procedures like chemical cauterization and fat plug myringoplasty have been found to be effective in healing small central perforations with significant hearing improvement.

Fat Plug Myringoplasty has many advantages compared with conventional myringoplasty using temporalis fascia, as it is short, simple, cost-effective and minimally invasive. Fat from ear lobule has been in use for last 15 20 years. Microscopic study of fat from ear lobule showed that the fat cells are more compact and contain more fibrous tissue. It provides scaffolding for the growth of tympanic membrane epithelium and mucus membrane by bridging the gap. In 1962, Ringenberg successfully used adipose tissue (Fat) to repair a small tympanic membrane perforation [2]. 


\section{Aims and Objectives}

1) To study the efficacy of chemical cauterization in small central perforations of tympanic membrane.

2) To study the efficacy of Fat Plug Myringoplasty in small central perforations of tympanic membrane.

3) To evaluate the outcome of these two procedures and compare them.

\section{Materials and Methods}

The study was carried out in the Department of ENT, Gauhati Medical College and Hospital, Guwahati for duration of 1 year from August 2011 to July 2012. The study group included patients above 15 years of age with central perforations of tympanic membrane up to $5 \mathrm{~mm}$ in size. The locations of the perforations in the pars tensa were variable from patient to patient as demonstrated in Figure 1. This study consisted of a total of 69 patients out of which 36 underwent Chemical Cauterization and 33 underwent Fat Plug Myringoplasty.

Criteria for patient selection:

1) Inclusion criteria:

a) Patients with central perforation of tympanic membrane following Chronic Suppurative Otitis Media or traumatic perforations of tympanic membrane.

b) At least 2 months old dry perforations were included.

c) Central perforations not more than $5 \mathrm{~mm}$ (35\%) by visual assessment were included in the study.

2) Exclusion criteria:

a) History of previous ear surgery;

b) Attico-antral disease;

c) Active discharge;

d) Nasopharyngeal pathology;

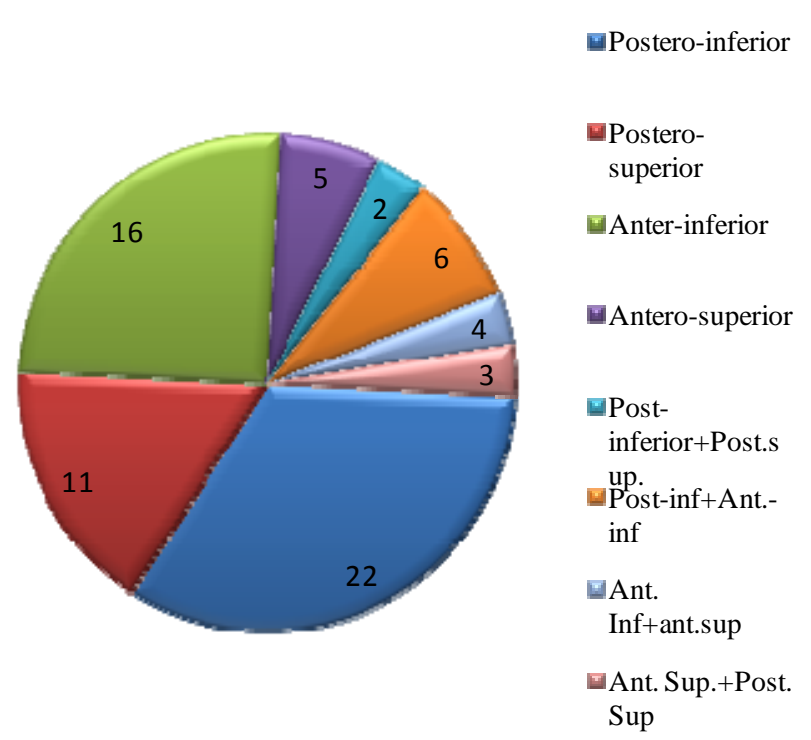

Figure 1. Pie diagram showing location of perforations in different quadrants of pars tensa. e) Eustachian tube dysfunction and;

f) Active rhinosinusitis.

Hearing assessment both before and after the procedures was done by Tuning fork tests and Pure Tone Audiometry.

Nasal Endoscopy was done to rule out any nasopharyngeal pathology, Eustachian tube dysfunction and rhinosinusitis etc.

\subsection{Chemical Cauterization (Paper Patching)}

1) Patients with central perforations less than $5 \mathrm{~mm}$ selected according to the section criteria mentioned above.

2) Patients were properly explained about the procedure and possible outcome and written and informed consent taken.

3) Cotton ball soaked in 4\% xylocaine was applied in the external auditory canal.

4) Under microscope, the edges of the tympanic membrane perforations were cauterized by using cotton tipped applicator dipped in trichloroacetic acid.

5) After the procedure, a thin paper preferably tissue paper was placed over the perforation followed by application of 1 - 2 drops of steroid containing antibiotic ear drop.

6) The perforation is inspected after 2 weeks and if required patient is called for subsequent sitting. A maximum of four applications were made.

7) After complete healing of the perforation the patient is followed up at 6 weeks and Pure Tone Audiometry was done to assess the hearing improvement.

\subsection{Fat Plug Myringoplasty}

1) After proper written and informed consent the concerned ear was prepared by betadine solution and then properly draped.

2) $2 \%$ xylocaine + adrenaline was infiltrated in the posterior aspect of the pinna and also in the external auditory canal.

3) Now by giving an incision in the posterior aspect of the ear lobule fat graft was harvested and the incision site was closed by 4 - 0 Ethilon.

4) Now under an operating microscope the edges of the tympanic membrane perforation were de-epithelialised (Figure 2).

5) The harvested fat plug is then wedged like a dumbbell one part of which is in the middle ear and other lateral to tympanic membrane then it is supported by a few pieces of gel foam from outside.

6) A course of antibiotic and anihistaminic drugs was given in the post-operative period

7) The patient was then followed up after 2 weeks, 6 weeks and then at 3 months and Pure Tone Audiometry was done to assess the hearing improvement (Figure 3). 


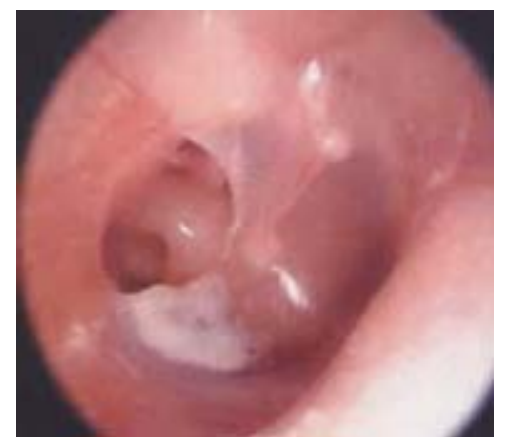

Figure 2. Central perforation (right tympanic membrane) of pars tensa.

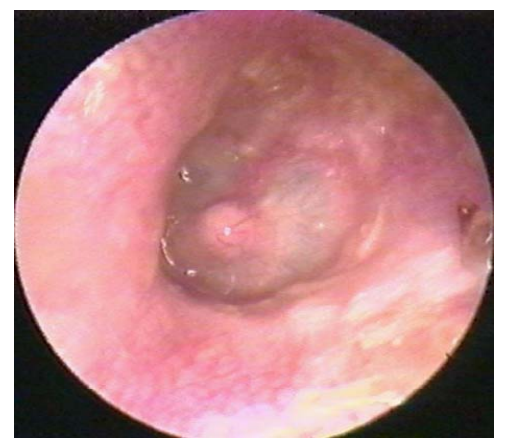

Figure 3. Healed right tympanic membrane perforation.

\subsection{Statistical Analysis}

The hearing gain after the two procedures was analyzed with Student's t-test, and the Pearson correlation coefficient (r) was determined by using Statistical Package for Social Science (SPSS) version 10.0. The other calculations performed for the present study was done by spreadsheets developed by Microsoft Excel (2007). A p value of 0.05 or less was considered significant for the study.

\section{Results and Observations}

The present study consisted of 69 patients out of which 40 were male and 29 female. The average age of the study group was 35 years. Out of 69 patients 30 patients had bilateral central perforations and 39 had unilateral perforations. 55 patients had perforations due to infective etiology and 14 had due to trauma. Out of 69 patients 36 underwent chemical cauterization and 33 underwent Fat Plug Myringoplasty.

In the group that underwent Chemical Cauterization the mean preoperative hearing threshold $(\mathrm{dB})$ was 24.66 \pm 3.69 and after the procedure the mean hearing threshold $(\mathrm{dB})$ was $16 \pm 2.03$ and there was a mean gain of about $8.66 \pm 3.69 \mathrm{~dB}$ (Figure 4) which was found to be statistically significant $(r=0.849, p=0.01)$. Out of 36 patients who underwent Chemical Cauterization 6 patients had residual perforations. The success rate was found to be $83.33 \%$.

In the group that underwent Fat Plug Myringoplasty the mean preoperative hearing threshold $(\mathrm{dB})$ was 33.83 \pm 4.41 and after the procedure the mean hearing threshold $(\mathrm{dB})$ was $17.83 \pm 2.84$ and there was a mean gain of about $16 \pm 4.02 \mathrm{~dB}$ (Figure 5) which was found to be statistically significant $(r=0.782, p=0.01)$. Out of 33 patients who underwent Fat Plug Myringoplasty 3 patients had residual perforations. The success rate was found to be $90.9 \%$.

Therefore, in our study we have found that Fat Plug Myringoplasty is a better option (success rate being 90.9\%) for the closure of Tympanic Membrane perforation less than $5 \mathrm{~mm}$ with only one surgical sitting. Whereas in Chemical Cauterization single application may not be sufficient in all cases.

In both the groups mentioned in the study, the number of patients having residual perforations (i.e. 6 out of 36 in Chemical Cauterization and 3 out of 33 in Fat Plug Myringoplasty) was not found to be statistically significant.

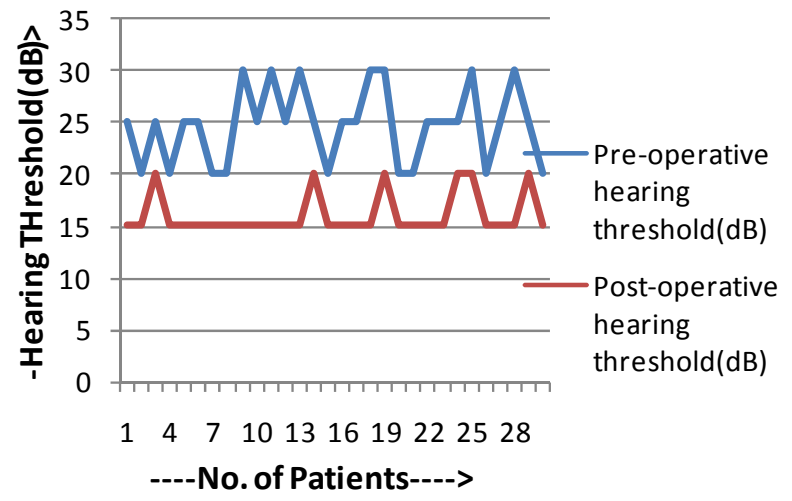

Figure 4. Line diagram showing comparison between pre and post operative hearing threshold $(\mathrm{dB})$ of patients undergoing chemical cauterization.

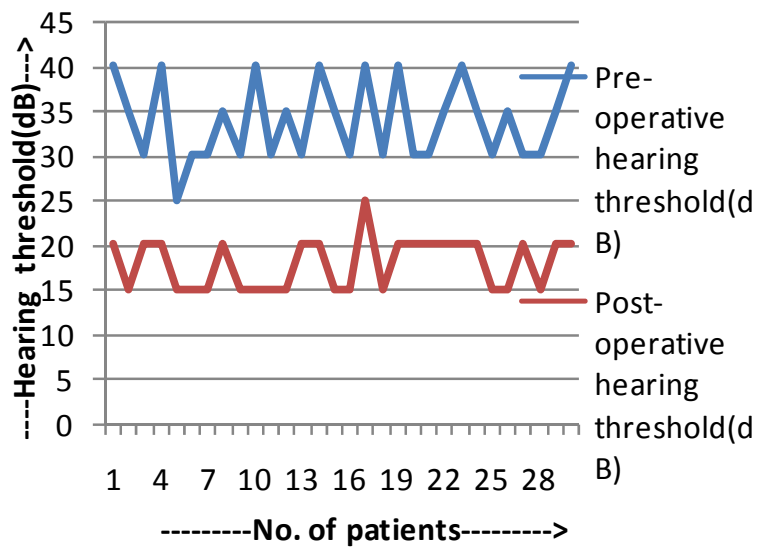

Figure 5. Line diagram showing comparison between pre and post-operative hearing threshold (dB) of patients undergoing fat plug myringoplasty. 


\section{Discussion}

The first recorded use of silver nitrate to stimulate closure of tympanic membrane perforations is by William Wilde in 1848 [3]. The office chemical myringoplasty was introduced by Roosa in 1876 and was popularized by Derlacki in the 1950s who reported good results and the procedure came to be known as the Derlacki method [4]. In his method he used trichloroacetic acid to cauterize perforation followed by antibiotic sufflation and covering with sterile cotton pledgets and prescribing Euthymol ear drops. Two decades later, Derlacki reported that he and his colleagues (Shambaugh, Harrison and Clemis) at Otologic Professional Associates at Chicago and cumulatively treated 1277 pars tensa perforations between 1953 and 1972 and had successfully healed at least 1027 of them (80.4\%) [5].

From our study it was found that the two procedures showed significant improvement in post operative hearing threshold. The overall success rate of $90.9 \%$ in patients undergoing Fat Plug Myringoplasty and 83.33\% in patients undergoing Chemical Cauterization observed in the present study is in agreement with the rates of $80 \%$ $92 \%$ reported in other studies. No significant difference in the tympanic membrane perforation closure rate was observed according to the perforation sites in different quadrants of the tympanic membrane.

Dursun E. et al. found out in 2008 that there were no statistically significant differences in tympanic membrane closure rates between paper patch, fat and perichondrium myringoplasty which is also found in our study [6].

Landsberg R. performed fat plug myringoplasty on a population comprising 27 adults and 11 children. The speech reception threshold improved significantly in his study. This is also seen in this study [7].

Nelson C. Goldman performed chemical cauterization in 11 patients and found $82.7 \%$ success rate [8]. Similar outcome is also seen in a study by Louis F. Scaramella $e t$ al. where success rate with chemical cauterization was 84.2\% which holds true in our study also [9].

Jadia S. et al. in their study found $92 \%$ and $88.8 \%$ outcome with fat plg myringoplasty and chemical cauterization which is also seen in this study [4].

Iqbal Hussain Udaipurwala et al. in 2008 in their study achieved 95.2\% success rate with Fat Plug Myringoplasty for central perforations of tympanic membrane which is consistent with this study [10].

Kuljit S. Uppal et al. in 1997 achieved 78\% success rate with chemical cautery of tympanic membrane perforations which is consistent with this study [11].

\section{Conclusion}

A reliable tympanic membrane closure rate in small central perforations was achieved with Fat Plug Myrin- goplasty and Chemical Cauterization without general anaesthesia in an official setting with a good amount of success. These two procedures are easy to perform, reliable with a satisfactory outcome, virtually simple and rapid risk free and also lessen the financial burden and morbidity on the part of the patient. Although both methods can be considered as convenient official procedures, the success rate is higher in Fat Plug Myringoplasty $(90.9 \%)$, and this procedure can be recommended as a better alternative/preferable choice for first line treatment of central perforations of the tympanic membrane in pars tensa less than $5 \mathrm{~mm}$ in size.

\section{Acknowledgements}

We are highly grateful to The Principal and the Superintendant of Gauhati Medical College and Hospital, Guwahati for allowing us to carry out the study in this Institution.

\section{REFERENCES}

[1] U. Chalishazar, “Fat Plug Myringoplasty,” Indian Journal of Otolaryngology and Head Neck Surgery, Vol. 57, No. 1, 2005, pp. 43-44.

[2] J. C. Ringerberg, "Closure of Tympanic Membrane Perforations by the Use of Fat," Laryngoscope, Vol. 88, No. 6, 1978, pp. 982-993. http://dx.doi.org/10.1288/00005537-197806000-00010

[3] W. R. Wilde and A. Hewson, "Practical Observations on Aural Surgery and the Nature and Treatment of Diseases of the Ear," Blanchard \& Lea, Philadelphia, 1853, pp. 292-293.

[4] S. Jadia, et al., "Office Procedure for the Management of Central Perforation: A Comparative Study,” Indian Journal of Otology, Vol. 16, 2010, pp. 5-9.

[5] E. L. Derlacki, "Office Closure of Central Tympanic Membrane Perforation: A Quarter Century Experience," Transactions of the American Academy of Ophthalmology, Vol. 77, 1973, pp. 53-56.

[6] E. Dursun, et al., "Comparison of Paper Patch, Fat and Perichondrium Myringoplasty in Repair of Small Tympanic Perforations,” Otolaryngology Head Neck Surgery, Vol. 138, No. 3, 2008, pp. 353-356. http://dx.doi.org/10.1016/j.otohns.2008.01.003

[7] R. Landsberg, G. Fishman, A. DeRowe, E. Berco and G. Berger, "Fat Graft Myringoplasty: Results of a LongTerm Follow-Up," Journal of Otolaryngology, Vol. 35, No. 1, 2006, pp. 44-47. http://dx.doi.org/10.2310/7070.2005.4124

[8] N. C. Goldman, "Chemical Closure of Chronic Tympanic Membrane Perforations,” ANZ Journal of Surgery, Vol. 77, No. 10, 2007, pp. 850-851. http://dx.doi.org/10.1111/j.1445-2197.2007.04256.x

[9] L. F. Scaramella, et al., "Effectiveness in Non-Surgical Office Closure of Tympanic Membrane Pars Tensa Perforations,” Ear, Nose and Throat Journal, Vol. 81, No. 8, 
2002, pp. 556-560.

[10] I. H. Udaipurwala, et al., "Efficacy of Fat-Plug Myringoplasty in Small and Dry Perforation of Pars Tensa," Medical Channel, Vol. 16, No. 1, 2010, pp. 62-64.
[11] K. S. Uppal, et al., "Closure of Tympanic Membrane Perforations by Chemical Cautery,” IJO \& HNS, Vol. 49, No. 2, 1997, pp. 151-153. 